\title{
FACTORIZATION OF TRIANGULAR OPERATORS AND IDEALS THROUGH THE DIAGONAL*
}

\author{
by JOHN LINDSAY ORR and DAVID R. PITTS
}

(Received 22nd March 1995)

\begin{abstract}
We give a necessary and sufficient condition to determine when an operator in the nest algebra of doubly infinite block upper triangular operators factors through a diagonal projection. An example shows that this condition does not extend to more general nest algebras, but a similar criterion yields a description of the ideals of nest algebras generated by diagonal projections.
\end{abstract}

1991 Mathematics subject classification: 47D25.

\section{Introduction}

Let $\mathcal{H}_{n}(n \in \mathbb{Z})$ be a sequence of infinite-dimensional Hilbert spaces, and let $\mathcal{H}:=\sum^{\oplus}\left\{\mathcal{H}_{n}: n \in \mathbb{Z}\right\}$. Write $E_{n}:=P\left(\mathcal{H}_{n}\right), P_{n}:=P\left(\cdots \oplus \mathcal{H}_{n-1} \oplus \mathcal{H}_{n}\right)$ where $P(S)$ is the orthogonal projection onto the subspace $S$ of $\mathcal{H}$. Let $\mathcal{N}$ be the nest in $\mathcal{H}$ consisting of the projections $P_{n}$ together with 0 and $I$.

The nest algebra, $\operatorname{Alg} \mathcal{N}$, is the set of doubly infinite block operator matrices. Let $D=\sum^{\oplus} D_{n}$ be a block diagonal projection, and $d_{n}=\operatorname{rank} D_{n}$. Since the projections $P_{n}$ are invariant for $\operatorname{Alg} \mathcal{N}$, and the intervals $P_{n}-P_{m}$ are semi-invariant, it follows that any operator $X$ which factors as $A D B$ for $A, B \in A \lg \mathcal{N}$ will satisfy:

$$
\operatorname{rank}\left(P_{n}-P_{m}\right) X\left(P_{n}-P_{m}\right) \leq \operatorname{rank}\left(P_{n}-P_{m}\right) D=\sum_{m<i \leq n} d_{i}
$$

for all $n \geq m$. The main technical result of this paper, Theorem 2.6 , shows that the converse also holds.

Our main application of this result is to describe the ideals in any nest algebra which are generated by diagonal projections (Theorem 3.1).

We were led to these results through an attempt to classify the ideals of continuous nest algebras. Ideals of nest algebras have been studied throughout the life of the subject, and in recent years, some very detailed information has been obtained about the ideals of continuous nest algebras. In particular, the family of maximal ideals [6] and the family of automorphism-invariant ideals [7] have both been classified.

*Both authors were partially supported by NSF grant DMS-9204811. 
The hope was (and still is) that the lattice of automorphism invariant ideals would provide a framework around which the whole lattice of ideals could be built up. We still believe this program is feasible, but the road appears more rocky than we anticipated.

The main obstacle is our inability to answer the following question: Let $\Delta$ be the conditional expectation of $\operatorname{Alg} \mathcal{N}$ onto its diagonal. Does $\Delta(X)$ belong to the ideal generated by $X$ for all $X \in A \lg \mathcal{N}$ ? If so, then the results of this paper, which show how to describe the ideal generated by $\Delta(X)$ would be immensely useful in understanding the general ideals of $\operatorname{Alg} \mathcal{N}$, and, probably, other nest algebras.

The obstacle described here is, moreover, one which has recently arisen in a number of questions on nest algebras (see the fuller discussion in $[3,6]$ ). A positive answer to this question would significantly deepen our understanding of nest algebras.

\section{Factorization through diagonal projections}

Definition 2.1. Let $\mathcal{N}$ be the nest described above and let $\left\{d_{i}: i \in \mathbb{Z}\right\}$ be a sequence of non-negative integers. We say that an operator $X$ is subordinate to the sequence $\left\{d_{i}: i \in \mathbb{Z}\right\}$ if

$$
\operatorname{rank}\left(P_{n}-P_{m}\right) X\left(P_{n}-P_{m}\right) \leq \sum_{m<i \leq n} d_{i} \text { for all } n \geq m
$$

Suppose that $D$ is a diagonal projection with rank $E_{i} D=d_{i}$ for all $i$. We observed above that every operator in $\operatorname{Alg} \mathcal{N}$ which factors through $D$ must be subordinate to $\left\{d_{i}\right\}$. In this section we shall prove the converse. To do this, we need to factor an operator which is subordinate to $\left\{d_{i}\right\}$ through another operator $A$ which satisfies rank $A E_{i} \leq d_{i}$ for all $i$. This is accomplished in Theorem 2.4, using the following lemma.

Lemma 2.2. Let $\left\{d_{i}\right\}$ be a sequence of non-negative integers indexed by $\mathbb{Z}$. Suppose that $X \in \operatorname{Alg} \mathcal{N}$ is subordinate to $\left\{d_{i}\right\}$ and the projection onto $\operatorname{Ker} X$ commutes with $\mathcal{N}$.

Then, for any fixed $N \in \mathbb{N}, X$ may be factored as $X^{\prime} U$, where $X^{\prime}$ and $U$ are operators in $\mathrm{Alg} \mathcal{N}$ satisfying:

(1) $U$ is a partial isometry whose initial space is the cokernel of $X$ and whose final space is the cokernel of $X^{\prime}$;

(2) $X^{\prime}$ is subordinate to $\left\{d_{i}\right\}$ and the projection onto $\operatorname{Ker} X^{\prime}$ commutes with $\mathcal{N}$;

(3) $\operatorname{rank} X^{\prime} E_{N} \leq d_{N}$.

Proof. The idea of the proof is to "shunt" part of the column operator $\left.X\right|_{\mathcal{H}_{N}}$ into $\mathcal{H}_{N-1}$. The trick is to do it in such a way that exactly the right part is left behind. To this end, we first construct the subspace $S$ on which the part, $\left.X\right|_{S}$, which will be left behind by the "shunting" will live.

For each $r \in \mathbb{Z}$, let 


$$
C_{r}:=\left\{x: P_{r}^{\perp} X E_{N} x \in \operatorname{Ran} P_{r}^{\perp} X P_{N-1}\right\} .
$$

Since our primary interest is in $C_{r}^{\perp}$, it is useful to observe that $C_{r}^{\perp}$ may be viewed as the largest part of the cokernel of $P_{r} X E_{N}$ which $P_{r} X E_{N}$ sends to a subspace independent of the range of $\left(P_{N-1}-P_{r}\right) X\left(P_{N-1}-P_{r}\right)$.

Observe that $\left\{C_{r}: r \in \mathbb{Z}\right\}$ is a chain of subspaces. For if $x \in C_{r}$ then $P_{r}^{\perp} X E_{N} x=$ $P_{r}^{\perp} X P_{N-1} y$ for some $y$. Multiplying on the left by $P_{r+1}^{\perp}$ shows that $x$ also belongs to $C_{r+1}$.

Notice that $C_{r}^{\perp}=(0)$ if $r \geq N$ and that $\operatorname{dim} C_{N-1}^{\perp} \leq d_{N}$ since

$$
C_{N-1}=\operatorname{ker} P_{N-1}^{\perp} X E_{N}=\operatorname{ker}\left(P_{N}-P_{N-1}\right) X\left(P_{N}-P_{N-1}\right) .
$$

Thus there are two cases. Either there is an $r<N$ such that

$$
\operatorname{dim} C_{r}^{\perp} \leq d_{N}<\operatorname{dim} C_{r-1}^{\perp}
$$

or else the sequence of dimensions, $\left\{\operatorname{dim} C_{r}^{\perp}\right\}_{r \in \mathcal{Z}}$, is bounded by $d_{N}$, in which case $C_{r}^{\perp}$ is finally constant as $r \rightarrow-\infty$. We shall deal with these two cases separately.

Case (i) In this case, we may choose $S$ to be a subspace of dimension exactly $d_{N}$, with

$$
C_{r_{0}}^{\perp} \subseteq S \subseteq C_{r_{0}-1}^{\perp}
$$

for some value of $r_{0}<N$.

Since the projection onto $\operatorname{Ker} X$ commutes with $\mathcal{N}$, the cokernel of $X$ is the direct sum of spaces $D_{n} \subseteq \mathcal{H}_{n}$. Clearly all the $C_{r}^{\perp}$ 's lie in $D_{N}$, so $S \subseteq D_{N}$. Let $U_{N}$ be a partial isometry which maps $D_{N} \ominus S$ and $D_{N-1}$ into $\mathcal{H}_{N-1}$, and set

$$
U:=U_{N}+P(S)+\sum_{n \neq N-1, N} P\left(D_{n}\right)
$$

Thus $U$ "shunts" part of $D_{N}$ into $H_{N-1}$ and acts as the identity on $S$ and all of the $D_{n}$ 's with $n \neq N-1, N$. So $U \in \operatorname{Alg} \mathcal{N}$, and the initial space of $U$ is the cokernel of $X$.

Define $X^{\prime}:=X U^{*}$. To show that $X^{\prime}$ has the required properties, we first note that the cokernel of $X^{\prime}$ is the final space of $U$, by construction, and that $X=X^{\prime} U$. Next, $X^{\prime} \in \operatorname{Alg} \mathcal{N}$ since $\operatorname{Ran} X^{\prime} P_{n}=\operatorname{Ran} X P_{n}$ for all $n \neq N-1$ and

$$
\begin{aligned}
\operatorname{Ran} X^{\prime} P_{N-1} & \subseteq \operatorname{Ran} X\left(P_{N-1}+P\left(D_{N} \ominus S\right)\right) \\
& \subseteq \operatorname{Ran}\left(P_{N-1}\right)+X C_{r_{0}} \\
& \subseteq \operatorname{Ran} P_{N-1} .
\end{aligned}
$$

It remains to show that $X^{\prime}$ is also subordinate to $\left\{d_{i}\right\}$. The compression of $X^{\prime}$ to $P_{n}-P_{m}$ has the same rank as the compression of $X$ to this projection unless one of $m$ 
or $n$ is equal to $N-1$. When $m=N-1$ then the compression of $X^{\prime}$ to $P_{n}-P_{N-1}$ equals the compression of $X\left(P(S)+P_{N}^{\perp}\right)$ to $P_{n}-P_{N-1}$ and all is well.

Suppose then that $n=N-1$ and consider the first case when $m \geq r_{0}$.

Then the range of the compression $\left(P_{N-1}-P_{m}\right) X^{\prime}\left(P_{N-1}-P_{m}\right)$ is

$$
\operatorname{Ran} P_{m}^{\perp} X P_{N-1}+\operatorname{Ran} P_{m}^{\perp} X\left(E_{N}-P(S)\right) \text {. }
$$

But

$$
\operatorname{Ran} P_{m}^{\perp} X\left(E_{N}-P(S)\right) \subseteq \operatorname{Ran} P_{m}^{\perp} X P\left(C_{r_{0}}\right) \subseteq \operatorname{Ran} P_{m}^{\perp} X P_{N-1}
$$

by the definition of the sets $C_{r}$, and so the rank of the compression of $X^{\prime}$ is dominated by the rank of the compression of $X$.

The final case, when $m<r_{0}$, is the most subtle. We claim that

$$
\begin{aligned}
\operatorname{rank}\left(P_{N-1}-\right. & \left.P_{m}\right) X^{\prime}\left(P_{N-1}-P_{m}\right) \\
& =\operatorname{rank}\left(P_{N}-P_{m}\right) X\left(P_{N}-P_{m}\right)-\operatorname{rank} P_{m}^{\perp} X P(S) \\
& \leq\left(d_{m+1}+\cdots+d_{N}\right)-\operatorname{dim} S \\
& =d_{m+1}+\cdots+d_{N-1},
\end{aligned}
$$

as desired. Since $S \subseteq C_{r_{0}-1}^{\perp} \subseteq C_{m}^{\perp}$ and $P_{m}^{\perp} X$ does not map (non-zero) vectors in $C_{m}^{\perp}$ into $\operatorname{Ran} P_{m}^{\perp} X P_{N-1}$, it follows that, in particular, $P_{m}^{\perp} X$ is one to one on $S$ and rank $P_{m}^{\perp} X P(S)=\operatorname{dim} S=d_{N}$. The equality claimed in line (1) will follow from the equation

$$
\operatorname{Ran}\left(P_{N}-P_{m}\right) X\left(P_{N}-P_{m}\right)=\operatorname{Ran} P_{m}^{\perp} X^{\prime} P_{N-1}+\operatorname{Ran} P_{m}^{\perp} X P(S)
$$

once we observe that the spaces $\operatorname{Ran} P_{m}^{\perp} X P(S)$ and $\operatorname{Ran} P_{m}^{\perp} X^{\prime} P_{N-1}$ are independent. Indeed, if $P_{m}^{\perp} X x \in \operatorname{Ran} P_{m}^{\perp} X^{\prime} P_{N-1}$ for some $x \in S$ then $P_{m}^{\perp} X x=P_{m}^{\perp} X(y+z)$ for some $y=P_{N-1} y$ and $z \in \mathcal{H}_{N} \ominus S$. But thus $P_{r_{0}-1}^{\perp} X(x-z) \in \operatorname{Ran} P_{r_{0}-1}^{\perp} X P_{N-1}$ and so $x-z \in C_{r_{0}-1} \subseteq S^{\perp}$. But since $z$ is also in $S^{\perp}$ and $x \in S$, this means that $x=0$ and we are done.

Case (ii) As $r$ decreases, the spaces $C_{r}^{\perp}$ increase. Since the dimensions of these spaces are bounded, they finally stabilize to a subspace $S$. Define $U$ and $X^{\prime}$ in the same way as above, but with this different choice of $S$. The argument from the first case is still adequate to show that $X=X^{\prime} U$, and that $X^{\prime}$ and $U$ belong to $\operatorname{Alg} \mathcal{N}$. The only part of the earlier argument which needs further scrutiny is the claim that $X^{\prime}$ is still subordinate to $\left\{d_{i}\right\}$. Once again, when we consider the compression to $\left(P_{n}-P_{m}\right)$, the only interesting case is when $n=N-1$. But then if we simply set $r_{0}:=m$, we can use the fact that this time $S^{\perp}$ is necessarily contained in $C_{r_{0}}$, to argue exactly as we did in the case when $m \geq r_{0}$ above.

Remark 2.3. Notice from the construction in the proof that $U$ commutes with all 
the $P_{n}$ 's except for $P_{N-1}$. By the same token, $X^{\prime} P_{N}^{\perp}=X P_{N}^{\perp}$ and $X^{\prime} P_{N-2}=X P_{N-2}$.

Theorem 2.4. Let $\left\{d_{n}\right\}$ be a sequence of non-negative integers indexed by $\mathbb{Z}$ and suppose that $X \in \operatorname{Alg} \mathcal{N}$ is subordinate to $\left\{d_{n}\right\}$. Then $X$ factors as $A B$ in $\operatorname{Alg} \mathcal{N}$, where rank $A E_{n} \leq d_{n}$ for all $n \in \mathbb{Z}$ and $\|A\|\|B\|=\|X\|$.

Proof. We first factor $X$ as $X_{0} V$ where $V \in \operatorname{Alg} \mathcal{N}$ is a partial isometry and $X_{0} \in \operatorname{Alg} \mathcal{N}$ satisfies the hypotheses of Lemma 2.2. The important thing is to ensure that the projection onto the cokernel of $X_{0}$ commutes with $\mathcal{N}$, while $X_{0}$ stays subordinate to $\left\{d_{i}\right\}$. This can be done using the inner-outer factorization of operators in nest algebras [1], but for completeness we present here a slightly less stringent factorization which is sufficient for our purposes. Let $Q$ be the projection onto the cokernel of $X$ and let $V$ be a partial isometry mapping the range of

$$
P_{n}^{\perp} \wedge Q-P_{n+1}^{\perp} \wedge Q
$$

into the range of $P_{n+1}-P_{n}$ for each $n$ and set $X_{0}:=X V^{*}$.

Clearly $V$ is in $\operatorname{Alg} \mathcal{N}$ and, since

$$
P_{n}^{\perp} X Q=P_{n}^{\perp} X=P_{n}^{\perp} X P_{n}^{\perp}
$$

it follows that the ranges of both $P_{n}^{\perp}$ and $Q$ contain the cokernel of $P_{n}^{\perp} X$. Thus $P_{n}^{\perp} X=P_{n}^{\perp} X\left(P_{n}^{\perp} \wedge Q\right)$ and so

$$
P_{n}^{\perp} X V^{*}=P_{n}^{\perp} X\left(P_{n}^{\perp} \wedge Q\right) V^{*}=P_{n}^{\perp} X V^{*} P_{n}^{\perp} .
$$

Thus $X V^{*}$ also belongs to $\operatorname{Alg} \mathcal{N}, X=\left(X V^{*}\right) V$ and the projection onto the cokernel of $X V^{*}$ commutes with $\mathcal{N}$.

Next we need to show that $X V^{*}$ is subordinate to $\left\{d_{i}\right\}$. Since both $X V^{*}$ and $V$ are in $\operatorname{Alg} \mathcal{N}$,

$$
\begin{aligned}
\left(P_{n}-P_{m}\right) X\left(P_{n}-P_{m}\right) & =\left(P_{n}-P_{m}\right)\left(X V^{*}\right) V\left(P_{n}-P_{m}\right) \\
& =\left(P_{n}-P_{m}\right) X V^{*}\left(P_{n}-P_{m}\right) V P_{n} .
\end{aligned}
$$

The compression condition for $X V^{*}$ then follows, because it turns out that the ranges of $\left(P_{n}-P_{m}\right) X V^{*}\left(P_{n}-P_{m}\right) V P_{n}$ and $\left(P_{n}-P_{m}\right) X V^{*}\left(P_{n}-P_{m}\right)$ are the same.

To see this, we need only show that the ranges of $V^{*}\left(P_{n}-P_{m}\right) V P_{n}$ and $V^{*}\left(P_{n}-P_{m}\right)$ are the same. But $V^{*}\left(P_{n}-P_{m}\right) V=P_{m}^{\perp} \wedge Q-P_{n}^{\perp} \wedge Q$ and so the range of this projection is disjoint from the range of $P_{n}^{\perp}$; hence $P_{n}$ is one to one on the range of $V^{*}\left(P_{n}-P_{m}\right) V$. It follows that

$$
\begin{aligned}
\operatorname{ker} P_{n} V^{*}\left(P_{n}-P_{m}\right) V & =\operatorname{ker} V^{*}\left(P_{n}-P_{m}\right) V \\
& =\operatorname{ker}\left(P_{n}-P_{m}\right) V
\end{aligned}
$$


and, taking adjoints, the closed ranges of $V^{*}\left(P_{n}-P_{m}\right) V P_{n}$ and $V^{*}\left(P_{n}-P_{m}\right)$ are the same. The ranges of these operators are closed (they are products of operators which take closed sets to closed sets) so their ranges are equal.

Now that we have $X$ factored as $X_{0} V$, we apply Lemma 2.2 repeatedly to $X_{0}$. First use the lemma to factor $X_{0}=X_{0,0} U_{0,0}$ where rank $X_{0,0} E_{0} \leq d_{0}$. Next, factor $X_{0,0}$ as $X_{1,1} U_{1,1}$ where rank $X_{1,1} E_{1} \leq d_{1}$ and then get $X_{1,0}$ by factoring $X_{1,1}=X_{1,0} U_{1,0}$ with rank $X_{1,0} E_{0} \leq d_{0}$, and $X_{1,-1}$ by then factoring $X_{1,0}=X_{1,-1} U_{1,-1}$ with rank $X_{1,-1} E_{-1} \leq d_{-1}$.

Proceed inductively in this way, obtaining $X_{n, n}$ by rolling over from the last level and factoring $X_{n-1,-(n-1)}=X_{n, n} U_{n, n}$ with rank $X_{n, n} E_{n} \leq d_{n}$. Then obtain $X_{n, k}$ by stepping down columns, from the $n$th to the $-n$ th; factoring $X_{n, k-1}$ as $X_{n, k} U_{n, k}$ subject to rank $X_{n, k} E_{k} \leq d_{k}$ at each stage.

The payoff from this is that, in view of the remark following the proof of Lemma 2.2, rank $X_{n, k} E_{\mathrm{i}} \leq d_{i}$ for $k \leq i \leq n$.

Now let $E:=P_{K}-P_{-K}$ be fixed. We claim that the range of $E X_{n, k} E$ always increases as we move through the $X_{n, k}$ 's in the order in which they were defined. For, applying Lemma 2.2 to the $k$ th column, $Y E_{k}$, of an operator $Y$ does not change the range of the compression to $E$ for any $k \neq K+1,-K+1$, since the right hand factor of $Y$ (i.e. the partial isometry) commutes with $E$. If we apply Lemma 2.2 to the $(K+1)$ st column, the range of $E Y^{\prime} E$ (the left hand factor of $Y$ ) might be greater than Ran $E Y E$, since part of $Y E_{K+1}$ may have been "shunted" into $Y^{\prime} E_{K}$, but it cannot be smaller. By the same token, the range of $Y^{\prime} E$ might be smaller than the range of $Y E$ when the lemma is applied to the $-K+1$ st column. But, since $Y^{\prime} \in A \lg \mathcal{N}$, the part of $Y E_{-K+1}$ which is "shunted" into $Y^{\prime} E_{-K}$ must map into $P_{-K}$. Thus, in fact, the ranges of $E Y E$ and $E Y^{\prime} E$ are the same in this case.

So, as we run through the successive factorizations which give rise to $X_{n, k}$, the ranges $\operatorname{Ran} E X_{n, k} E$ stay constant except for a possible increase each time $k$ moves from $K+2$ to $K+1$ (and, exactly once, when we roll over from $X_{K,-K}$ to $X_{K+1, K+1}$ ).

Also, of course, since $X_{n, k}$ remains subordinate to $\left\{d_{i}\right\}$ through all of these factorizations, rank $E X_{n, k} E$ is bounded above by $\sum_{-K}^{K} d_{i}$. Thus the range of $E X_{n, k} E$ is finally constant. Moreover, the projection onto the cokernel of $X_{n, k}$ commutes with $\mathcal{N}$, so we can modify $X_{n, k}$ and $U_{n, k}$ by multiplying by a diagonal unitary to suppose in addition that the cokernels of $E X_{n, k} E$ are finally constant. Now, write $Y_{n}$ for $X_{n_{1}-n}$ and $W_{n}$ for the product

$$
U_{n,-n} U_{n,-n+1} \cdots U_{2,2} U_{1,-1} U_{1,0} U_{1,1} U_{0,0}
$$

and bear in mind that $X_{0}=Y_{n} W_{n}$.

We have seen that $E Y_{n} E$ is a bounded sequence of operators with fixed finite dimensional range and cokernel. Thus it has a norm-convergent subsequence. Throughout this discussion $E$ was fixed, but by a diagonal argument we can pick a subsequence $Y_{n(i)}$ so that $E Y_{n(i)}$ converges for each interval $E=P_{K}-P_{-K}$. Further, passing to a subnet, we may suppose that $W_{n(i)}$ and $Y_{n(i)}$ converge weakly, say to $X^{\prime}$ and $W^{\prime}$. Then for any fixed $E=P_{K}-P_{-K}$, 


$$
\begin{aligned}
E X^{\prime} W^{\prime} E & =\left(E X^{\prime} E\right)\left(E W^{\prime} E\right) \\
& =\text { norm-lim }\left(E Y_{n(i)} E\right) \text { weak-lim }\left(E W_{n(i)} E\right) \\
& =\text { weak-lim }\left(E Y_{n(i)} W_{n(i)}\right) \\
& =E X_{0} E .
\end{aligned}
$$

If we now let $E$ increase to the identity, we see that $X_{0}=X^{\prime} W^{\prime}$. Moreover, for each $k$, rank $X_{n(1)} E_{k} \leq d_{k}$ eventually, so rank $X^{\prime} E_{k} \leq d_{k}$. Thus $X=X^{\prime}\left(W^{\prime} V\right)$ is the desired factorization.

Remark 2.5. Because $W^{\prime}$ is only the weak limit of partial isometries we can only conclude from this argument that it is a contraction. We expect a factorization by a partial isometry is possible, but we do not have a proof.

A nest is called discrete if it has no limit points other than, possibly, 0 or $I$. Discrete nests are ordered as one of

$$
\mathbb{Z} \cup\{-\infty,+\infty\}, \mathbb{N} \cup\{+\infty\},-\mathbb{N} \cup\{-\infty\}, \quad \text { or a finite set. }
$$

Theorem 2.6. Let $\mathcal{M}$ be a discrete nest acting on a Hilbert space $\mathcal{H}$ and let $P$ be a projection in the diagonal of $\operatorname{Alg} \mathcal{M}$. Then $X \in \operatorname{Alg} \mathcal{M}$ factors as $X=A P B$ in $\operatorname{Alg} \mathcal{M}$ if and only if

$$
\operatorname{rank}(N-M) X(N-M) \leq \operatorname{rank}(N-M) P
$$

for all $N>M$ in $\mathcal{M}$.

Proof. First, suppose that the compressions of $P$ to the atoms of $\mathcal{M}$ are all finite dimensional.

It is straightforward to construct a Hilbert space $\mathcal{K} \supseteq \mathcal{H}$ together with a nest $\mathcal{N}=\left\{P_{n}: n \in \mathbb{Z}\right\} \cup\{0, I\}$ on $\mathcal{K}$ such that the projection $Q$ of $\mathcal{K}$ onto $\mathcal{H}$ commutes with $\mathcal{N}, \mathcal{M}=Q \mathcal{N}$, and the atoms of $\mathcal{N}$ are all infinite rank. Let $d_{n}=\operatorname{rank}\left(P_{n}-P_{n-1}\right) P$, which is a sequence of non-negative integers.

Clearly $X$ is subordinate to $\left\{d_{n}\right\}$ along $\mathcal{N}$, so by Theorem 2.4 there are $A, B$ in $\operatorname{Alg} \mathcal{N}$ factoring $X$, and with

$$
\operatorname{rank} A\left(P_{n}-P_{n-1}\right) \leq d_{n} \text {. }
$$

Let $V$ be a partial isometry which commutes with $\mathcal{N}$ and which maps the cokernel of each column $A\left(P_{n}-P_{n-1}\right)$ to a subspace of $\left(P_{n}-P_{n-1}\right) P$. Then

$$
X=\left(Q A V^{*}\right) P(V B Q)
$$

is a factorization in $\operatorname{Alg} \mathcal{M}$. 
In the general case, some compressions of $P$ to atoms of $\mathcal{M}$ may be infinite dimensional. Let $P_{\infty}$ be the join of all those atoms of $\mathcal{M}$ which meet $P$ in infinite rank projections. Decompose $I-P_{\infty}$ as the sum of its maximal subintervals $E_{i}=M_{i}-N_{i}$ (indexed in no particular order), which are necessarily pairwise orthogonal. Given $X \in \operatorname{Alg} \mathcal{M}$ which satisfies the compression conditions, our first job is to factor $X_{\infty}:=X-\sum_{i} E_{i} X E_{i}$ through $P_{\infty}$.

For each $i$, unless $M_{i}=I, M_{i}$ has an immediate successor, $M_{i}^{+}$and $\left(M_{i}^{+}-M_{i}\right) P$ has infinite rank. Take $V$ to be a partial isometry which maps each interval $M_{i}^{+}-N_{i}$ (for $\left.M_{i} \neq I\right)$ into a subspace of $\left(M_{i}^{+}-M_{i}\right) P$ of infinite codimension, and which maps each atom $E$ for which $E P$ has infinite rank into a subspace of $E P$ of infinite codimension. Notice that $V^{*} \in \operatorname{Alg} \mathcal{M}$. We likewise construct a partial isometry $W \in \operatorname{Alg} \mathcal{M}$ which maps the intervals $\left(M_{i}-N_{i}^{-}\right)$into $N_{i}-N_{i}^{-}$(where $N_{i}^{-}$is the immediate predecessor which every $N_{i} \neq 0$ has) and likewise maps each atom $E$ for which $E P$ has infinite rank into a subspace of $E P$ having infinite codimension. In addition, arrange that the ranges of $V$ and $W$ are mutually orthogonal.

Then, if there is an $M_{i}=I$, write $N$ for the corresponding $N_{i}$, otherwise take $N=I$. It follows that $V^{*} V=N$ and $W^{*} W \geq N^{\perp}$. Thus we have

$$
X_{\infty}=V^{*}\left(V N X_{\infty}\right)+\left(N^{\perp} X_{\infty} W^{*}\right) W=\left(V^{*}+N^{\perp} X_{\infty} W^{*}\right)\left(V N X_{\infty}+W\right)
$$

which factors $X_{\infty}$ through $P_{\infty}$. The fact that $X_{\infty} M_{i}=N_{i} X_{\infty} M_{i}$ ensures that $V N X_{\infty}$ and $N^{\perp} X_{\infty} W^{*}$ belong to $\operatorname{Alg} \mathcal{M}$.

Next, we factor $X_{f}:=\sum E_{i} X E_{i}$ through $P_{f}:=I-P_{\infty}$. To do this, simply apply the argument from the first two paragraphs of this proof to $X_{f}$ in the nest algebra $P_{f}(\operatorname{Alg} \mathcal{M}) P_{f}=\operatorname{Alg} P_{f} \mathcal{M}$ to get $X_{f}=A P_{f} B$. To conclude the proof, observe that we have factored $X_{\infty}$ as $A^{\prime} P_{\infty} B^{\prime}$, so that

$$
X=X_{\infty}+X_{f}=A^{\prime} P_{\infty} B^{\prime}+A P_{f} B=\left(A^{\prime} P_{\infty}+A P_{f}\right) P\left(P_{\infty} B^{\prime}+P_{f} B\right)
$$

Unfortunately, Theorem 2.6 does not hold for more general nests. In the next example, we exhibit a nest $\mathcal{M}$ and a diagonal projection $P$ for which there is an operator $X \in A \lg \mathcal{M}$ which satisfies

$$
\operatorname{rank}(N-M) X(N-M) \leq \operatorname{rank}(N-M) P
$$

for all $N>M$ in $\mathcal{M}$, but which does not factor through $P$.

Example 2.7. Let $\mathcal{M}$ be an atomic nest with order type $\omega+\omega^{*}$ and infinite rank atoms. That is to say $\mathcal{M}$ consists of a projection $M$ together with two chains $M_{n}$ and $N_{n}(n \in \mathbb{N})$ of projections where $M_{n}$ increases from 0 to $M$ and $N_{n}$ decreases from $I$ to $M$. Let $P$ be a diagonal projection with

$$
\operatorname{rank} P\left(M_{n+1}-M_{n}\right)=\operatorname{rank} P\left(N_{n}-N_{n+1}\right)=1
$$


for all $n$. Then clearly any operator of the form $X=P+X_{0}$ with $X_{0} \in M B(\mathcal{H}) M^{\perp}$ will satisfy the rank condition of Theorem 2.6 in comparison with $P$. Yet we claim that an operator of this form only factors through $P$ if $P^{\perp} X_{0} P^{\perp}=0$.

For, writing $P_{1}:=P M$ and $P_{2}:=P M^{\perp}$, then $X$ factors through $P$ in $\operatorname{Alg} \mathcal{M}$ if and only if

$$
\begin{aligned}
\left(\begin{array}{cc}
P_{1} & X_{0} \\
0 & P_{2}
\end{array}\right) & =\left(\begin{array}{cc}
A_{11} & A_{12} \\
0 & A_{22}
\end{array}\right)\left(\begin{array}{cc}
P_{1} & 0 \\
0 & P_{2}
\end{array}\right)\left(\begin{array}{cc}
B_{11} & B_{12} \\
0 & B_{22}
\end{array}\right) \\
& =\left(\begin{array}{cc}
A_{11} P_{1} B_{11} & A_{11} P_{1} B_{12}+A_{12} P_{2} B_{22} \\
0 & A_{22} P_{2} B_{22}
\end{array}\right)
\end{aligned}
$$

for suitable operators in $\operatorname{Alg} \mathcal{N}$. But thus $\operatorname{rank}\left(M_{n+1}-M_{n}\right) A_{11} P_{1} B_{11}\left(M_{n+1}-M_{n}\right)=$ $\operatorname{rank}\left(M_{n+1}-M_{n}\right) P_{1}=1$. Since $\left(M_{n+1}-M_{n}\right)$ is a semi-invariant projection, this means that $\left(M_{n+1}-M_{n}\right) P_{1} B_{11}\left(M_{n+1}-M_{n}\right)$ is non-zero for all $n$ and so $\operatorname{Ran} P_{1} B_{11}$ is dense in Ran $P_{1}$. Thus

$$
\operatorname{Ran} A_{11} P_{1} B_{12} \subseteq \overline{\operatorname{Ran}} A_{11} P_{1}=\overline{\operatorname{Ran}} A_{11} P_{1} B_{11}=\operatorname{Ran} P_{1}
$$

By the same token, the cokernel of $A_{12} P_{2} B_{22}$ is contained in Ran $P_{2}$, and so

$$
P_{1}^{\perp}\left(A_{11} P_{1} B_{12}+A_{12} P_{2} B_{22}\right) P_{2}^{\perp}=0
$$

which yields $P^{\perp} X P^{\perp}=0$.

\section{Ideals generated by diagonal projections}

Although the factorization result of Theorem 2.4 does not hold for general nests, we can use it to describe the algebraic (not necessarily norm-closed) ideal generated by an arbitrary diagonal projection in any nest algebra.

Theorem 3.1. Let $\mathcal{N}$ be a nest and let $P$ be a projection in the diagonal of $\mathrm{Alg} \mathcal{N}$. Then the set of operators $X$ in Alg $\mathcal{N}$ for which there is a constant $K$ such that

$$
\operatorname{rank}(N-M) X(N-M) \leq K \operatorname{rank}(N-M) P
$$

for all $N>M$ in $\mathcal{N}$ coincides with the algebraic ideal generated by $P$.

Proof. For each $N \in \mathcal{N}$ let $U(N)$ be the join of all $M \geq N$ in $\mathcal{N}$ such that $(M-N) P$ has finite rank. Similarly, $L(N)$ is the meet of all $M \leq N$ such that $(N-M) P$ has finite rank. The distinct non-zero intervals of the form $U(N)-L(N)$ are pairwise orthogonal, so we can enumerate them as $E_{i}$.

Let $\mathcal{I}_{P}$ denote the algebraic ideal generated by $P$. Given $X \in A \lg \mathcal{N}$ which satisfies 
the rank condition, we first show that $\sum_{i} E_{i} X E_{i} \in \mathcal{I}_{p}$. Let $n$ be a positive integer greater than $K$ and let $Q$ be a rank-one projection in $M_{n}(\mathbb{C})$. Fix $i$ and let $X_{i}:=E_{i} X E_{i} \otimes Q, P_{i}:=P E_{i} \otimes I_{n}$ and let $\mathcal{N}_{i}:=E_{i} \mathcal{N} \otimes I_{n}$.

It is easy to see that the compression condition for $X$ implies that each $X_{i}$ satisfies the compression condition of Theorem 2.6 with respect to $P_{i}$ in $\operatorname{Alg} \mathcal{N}_{i}$. However, since $\mathcal{N}_{i}$ need not be a discrete nest, we cannot apply Theorem 2.6 directly in $\operatorname{Alg} \mathcal{N}_{i}$.

To deal with this, let $C_{i}$ be the central cover of $P_{i}$ in the diagonal of $\operatorname{Alg} \mathcal{N}_{i}$. Then $C_{i}$ is a sum of atoms of $\mathcal{N}_{i}$ and the set of end points of these atoms is ordered as a subset of the integers. Let $\mathcal{L}_{i}$ be the nest consisting of the endpoints of these atoms, together with the endpoints of $E_{i}$, so that $\mathcal{L}_{i}$ is a discrete subnest of $\mathcal{N}_{i}$. Then $X_{i}$ satisfies the conditions of Theorem 2.6 to factor through $P_{i}$ in $\operatorname{Alg} \mathcal{L}_{i}$ and

$$
X_{i}=A_{i} P_{i} B_{i}=\left(A_{i} C_{i}\right) P_{i}\left(C_{i} B_{i}\right)
$$

Since $C_{i}$ is a sum of atoms of $\mathcal{N}_{i}$, both $A_{i}=A_{i} C_{i}$ and $B_{i}=C_{i} B_{i}$ belong to $\operatorname{Alg} \mathcal{N}_{i}=\left(E_{i} \operatorname{Alg} \mathcal{N} E_{i}\right) \otimes M_{n}(\mathbb{C})$.

Pick partial isometries $V_{j}(1 \leq j \leq n)$ in $M_{n}(\mathbb{C})$ with pairwise orthogonal ranges and $V_{j}^{*} V_{j}=Q$. Then

$$
E_{i} X E_{i} \otimes Q=\sum_{1 \leq j \leq n}(I \otimes Q) A_{i}\left(I \otimes V_{j}\right)(I \otimes Q) P_{i}(I \otimes Q)\left(I \otimes V_{j}^{*}\right) B_{i}(I \otimes Q)
$$

Thus $E_{i} X E_{i}$ can be expressed as the sum $\sum_{j=1}^{n} A_{i, j} P E_{i} B_{i, j}$, where $A_{i, j}, B_{i, j} \in \operatorname{Alg} E_{i} \mathcal{N}$ and $\left\|A_{i, j}\right\|,\left\|B_{i, j}\right\| \leq\|X\|^{1 / 2}$. Regarding $A_{i, j}$ and $B_{i, j}$ as operators in $\operatorname{Alg} \mathcal{N}$, set $S_{j}:=\sum_{i=1}^{\infty} A_{i, j}$ and $T_{j}:=\sum_{i=1}^{\infty} B_{i, j}$ and note that

$$
\sum_{i} E_{i} X E_{i}=\sum_{j=1}^{n}\left(\sum_{i} A_{i j}\right)\left(\sum_{i} E_{i} P\right)\left(\sum_{i} B_{i j}\right)=\sum_{j=1}^{n} S_{j} P T_{j}
$$

It remains to show that $X_{2}:=X-\sum E_{i} X E_{i}$ belongs to the ideal generated by $P$. We shall need the following result, which is a simple rephrasing of Proposition 4.3 of [7].

Proposition 3.2. Let $\mathcal{N}_{\mathrm{c}}$ be a continuous nest and $E_{i}$ a sequence of pairwise orthogonal intervals of $\mathcal{N}_{c}$. Suppose that $T \in \operatorname{Alg} \mathcal{N}_{c}$ satisfies $\|E T E\| \geq 1$ whenever $E$ is an interval of $\mathcal{N}_{c}$ which is not dominated by any $E_{i}$. Then every operator $Y \in \operatorname{Alg} \mathcal{N}_{c}$ with

$$
E_{i} Y E_{i}=0 \quad \text { for all } i
$$

factors through $T$.

Now let $\mathcal{N}_{\infty}:=\mathcal{N} \otimes I$, where $I$ is the identity on an infinite dimensional Hilbert space, $\mathcal{K}$, let $Q$ be a rank-one projection on $\mathcal{K}$, and $P_{0}:=P \otimes Q$. Since all the atoms of $\mathcal{N}_{\infty}$ are infinite rank, we can find a continuous nest $\mathcal{N}_{c}$ containing $\mathcal{N}_{\infty}$. 
If we can find $A$ and $B$ in $\operatorname{Alg} \mathcal{N}_{\infty}$ so that $T:=A P_{0} B$ belongs to $\operatorname{Alg} \mathcal{N}_{c}$ and satisfies:

$\|E T E\| \geq 1$ for any interval $E$ of $\mathcal{N}_{c}$ which is not dominated by any $E_{i} \otimes I$,

then by the last proposition, $X_{2} \otimes Q$ factors through $T$ in $\operatorname{Alg} \mathcal{N}_{c} \subseteq \operatorname{Alg} \mathcal{N}_{\infty}$ and so

$$
X_{2} \otimes Q=(I \otimes Q) A^{\prime} A(P \otimes Q) B B^{\prime}(I \otimes Q),
$$

which gives a factorization of $X_{2}$ in $\operatorname{Alg} \mathcal{N}$.

To accomplish this, let $F_{i}$ be an enumeration of the atoms of $\mathcal{N}_{\infty}$. If $F_{i} P_{0}$ is infinite rank, then let $B_{i}$ be an isometry of $F_{i}$ into $F_{i} P_{0}$, and let $A_{i}=B_{i}^{*}$. If $F_{i} P_{0}$ is non-zero but finite rank, just choose $A_{i}, B_{i}$ to be any partial isometries on $F_{i}$ such that $A_{i} F_{i} P_{0} B_{i}$ is norm 1 and belongs to $\operatorname{Alg} \mathcal{N}_{c}$. Let $A:=P_{c}+\sum_{i} A_{i}$ and $B:=P_{c}+\sum_{i} B_{i}$, where $P_{c}:=I-\sum_{i} F_{i}$ is the projection onto the continuous part of $\mathcal{N}_{\infty}$. Then $T:=A P_{0} B$ satisfies $\|E T E\|=1$ when $E$ is any interval of $\mathcal{N}_{\infty}$ for which $E P_{0} \neq 0$.

Now suppose $E$ is an interval of $\mathcal{N}_{c}$ which is not dominated by any $E_{i} \otimes I$. We can decompose $E$ as a sum $E^{\prime}+E^{\prime \prime}+E^{\prime \prime \prime}$ of intervals of $\mathcal{N}_{c}$, where $E^{\prime}$ and $E^{\prime \prime \prime}$ are dominated by atoms of $\mathcal{N}_{\infty}$, and $E^{\prime \prime}$ is itself an interval of $\mathcal{N}_{\infty}$. If no $E_{i} \otimes I$ dominates $E^{\prime \prime}$ then $E^{\prime \prime} P_{0} \neq 0$ so $\|E T E\|=1$. On the other hand, if a certain $E_{i_{0}} \otimes I \geq E^{\prime \prime}$ then, by the construction of the $E_{i}$ 's, no other $E_{i} \otimes I$ can dominate either $E^{\prime}$ or $E^{\prime \prime \prime}$ (provided they are non-zero). Since $E_{i_{0}} \otimes I$ does not dominate $E$, it cannot dominate both of $E^{\prime}$ and $E^{\prime \prime \prime}$; so say it does not dominate $E^{\prime}$. Then in fact no $E_{i} \otimes I$ dominates $E^{\prime}$ and so likewise no $E_{i} \otimes I$ dominates the atom $F^{\prime}$ of $\mathcal{N}_{\infty}$ which contains $E^{\prime}$. This tells us that $F^{\prime} P_{0}$ has infinite rank and so $E T E=E F^{\prime}=E^{\prime} \neq 0$ and so $E T E$ is norm 1 .

Corollary 3.3. Let $\mathcal{N}$ be a nest and let $E$ be an idempotent in $\operatorname{Alg} \mathcal{N}$. Then the set of operators $X$ in $\operatorname{Alg} \mathcal{N}$ for which there is a constant $K$ such that

$$
\operatorname{rank}(N-M) X(N-M) \leq K \operatorname{rank}(N-M) E(N-M)
$$

for all $N>M$ in $\mathcal{N}$ coincides with the algebraic ideal generated by $E$.

Proof. Suppose that $E$ is an idempotent in $\operatorname{Alg} \mathcal{N}$. Letting $S$ be the positive square root of $\left(E^{*} E+(I-E)^{*}(I-E)\right)$, we find that $S E S^{-1}$ is a self-adjoint projection in $\operatorname{Alg} S \mathcal{N}$ where $S \mathcal{N}:=\{[S N]: N \in \mathcal{N}\}$. The proof of Lemma 2.1 of [5] shows that for any operator $Y \in \operatorname{Alg} \mathcal{N}$,

$$
\operatorname{rank}([S N]-[S M]) S Y S^{-1}([S N]-[S M])=\operatorname{rank}(N-M) Y(N-M) .
$$

This equality shows that $S$ conjugates the set of operators satisfying the conditions of this corollary onto the set of operator satisfying the conditions of Theorem 3.1 for $S E S^{-1}$. Since this latter set is the algebraic ideal generated by $S E S^{-1}$, the result follows. 
Corollary 3.4. Let $P_{1}, \ldots, P_{n}$ be projections in the nest algebra $\mathrm{Alg} \mathcal{N}$. Then the algebraic ideal generated by the $P_{i}$ is the set of operators $X \in A \lg \mathcal{N}$ with the property that there is a $K$ such that for all intervals $E$ if $\mathcal{N}$,

$$
\operatorname{rank} E X E \leq K \max _{i} \operatorname{rank} E P_{i} E
$$

Proof. The ideal generated by the $P_{i}$ is generated by $P_{1} \vee \cdots \vee P_{n}$, and

$$
\operatorname{rank} E\left(P_{1} \vee \cdots \vee P_{n}\right) E \leq \max E P_{i} E
$$

Theorem 3.1 provides a little information about the norm closed ideal generated by a general diagonal operator. If $D$ is in the diagonal of $\operatorname{Alg} \mathcal{N}$, we can express $|D|$ as the spectral integral $\int_{0}^{\infty} x d E_{x}$. The closed ideal generated by $D$ is the same as the closed ideal generated by the spectral projections $\left\{E_{x}: x>0\right\}$. Clearly

$$
\left\{X \in \operatorname{Alg} \mathcal{N}: \exists K, x>0 \text { such that } \forall \text { intervals } E \text {, rank } E X E \leq K \operatorname{rank} E E_{x}\right\}
$$

is dense in the ideal generated by $D$. This is not a very effective description unless one can tell whether a particular operator lies in the closure of this set.

Unfortunately, we are unable to describe the norm closed ideals generated by projections any more satisfactorily than to characterize them as the norm closures of the sets of operators described in Theorem 3.1. It sometimes happens, though, that the algebraic ideal generated by a particular projection (which we can describe) turns out to be weakly closed. This happens whenever the projection is a finite sum of intervals of the nest and in many other cases too. (To give another example, if $\operatorname{Alg} \mathcal{N}$ is the algebra of all infinite upper triangular matrices with respect to a fixed basis, and $P$ is the projection onto the even numbered basis elements then the ideal generated by $P$ consists of all matrices whose even numbered diagonal positions are zero. This is clearly weakly closed.) A weakly closed ideal generated by a projection has the following particularly nice description which is an easy consequence of Corollary 1.7 of [4]:

$$
\left\{X \in \operatorname{Alg} \mathcal{N}: E_{i} X E_{i}=0 \text { for all } i\right\}
$$

where $E_{i}$ is a sequence of pairwise orthogonal intervals of $\mathcal{N}$. Moreover, if the ideal generated by a projection is even norm closed, it is automatically weakly closed, as the next result shows.

Theorem 3.5. Let $P$ be a projection in the nest algebra $\operatorname{Alg} \mathcal{N}$. The algebraic ideal generated by $P$ in $\mathrm{Alg} \mathcal{N}$ is norm closed if and only if it is weakly closed.

Proof. Suppose that the algebraic ideal, $\mathcal{I}$, generated by $P$ is norm-closed and let $X$ be a fixed operator in the weak closure of $\mathcal{I}$. The aim is to construct an operator $Y$ as a norm-convergent sum of operators in $\mathcal{I}$, with the property that rank $E X E \leq 2$ rank $E Y E$ for all intervals $E$ of $\mathcal{N}$. Then, since $Y$ belongs to $\mathcal{I}$ and so 
satisfies the criterion of Theorem 3.1, the same is true of $X$. We shall make use of the weak operator topology lower semicontinuity of the map $A \mapsto \operatorname{rank} A$, together with the fact that

$$
\max \{\operatorname{rank} A+B, \operatorname{rank} A-B\} \geq \frac{1}{2} \max \{\operatorname{rank} A, \operatorname{rank} B\}
$$

which is a straightforward consequence of the subadditivity of $A \mapsto \operatorname{rank} A$.

Nests on separable Hilbert spaces are separable in the weak operator topology, so we can find a countable set of intervals with the property that every interval of $\mathcal{N}$ is the limit of an increasing sequence from this set. Enumerate these intervals as $\left\{E_{i}: i \in \mathbb{N}\right\}$ in such a way that each individual interval is listed infinitely many times. By the weak lower semicontinuity of the rank, we can pick contractions $X_{n} \in \mathcal{I}$ such that

$$
\operatorname{rank} E_{n} X_{n} E_{n}= \begin{cases}\operatorname{rank} E_{n} X E_{n}, & \text { if rank } E_{n} X E_{n} \text { is finite; } \\ n, & \text { if rank } E_{n} X E_{n} \text { is infinite }\end{cases}
$$

Next, we shall inductively choose a sequence $\left\{a_{k}\right\}$ of positive numbers and $\varepsilon_{k} \in\{-1,1\}$ to force convergence in norm of $\sum_{k} \varepsilon_{k} a_{k} X_{k}$. Start with $a_{1}=\varepsilon_{1}=1$. Then, with $a_{k}, \varepsilon_{k}$ chosen for $k=1, \ldots, n-1$, find a $0<\delta_{n}<1$ such that

$$
\operatorname{rank}\left(Z+E_{n-1} \sum_{k=1}^{n-1} \varepsilon_{k} a_{k} X_{k} E_{n-1}\right) \geq \operatorname{rank}\left(E_{n-1} \sum_{k=1}^{n-1} \varepsilon_{k} a_{k} X_{k} E_{n-1}\right)
$$

for all operators $Z$ with $\|Z\|<\delta_{n}$. (This is possible since the weak lower semicontinuity of the rank tells us that $\operatorname{rank}(A+Z) \geq \operatorname{rank} A$ for all sufficiently small $Z$.) Set

$$
a_{n}=\frac{a_{n-1} \delta_{n}}{2^{n}}
$$

and then pick $\varepsilon_{n} \in\{-1,1\}$ so that

$$
\operatorname{rank}\left(E_{n} \sum_{k=1}^{n} a_{k} \varepsilon_{k} X_{k} E_{n}\right) \geq \frac{1}{2} \operatorname{rank} E_{n} X_{n} E_{n}
$$

Notice that $\sum_{k=n}^{\infty} a_{n}<\delta_{n}$ for all $n$, and so, in particular, $Y:=\sum_{k=1}^{\infty} \varepsilon_{k} a_{k} X_{k}$ belongs to $\mathcal{I}$.

Let $E_{n}$ be fixed. Since $\left\|\sum_{k=n+1}^{\infty} \varepsilon_{k} a_{k} X_{k}\right\|<\delta_{n+1}$.

$$
\begin{aligned}
\operatorname{rank} E_{n} \sum_{k=1}^{\infty} \varepsilon_{k} a_{k} X_{k} E_{n} & \geq \operatorname{rank} E_{n} \sum_{k=1}^{n} \varepsilon_{k} a_{k} X_{k} E_{n} \\
& \geq \frac{1}{2} \operatorname{rank} E_{n} X_{n} E_{n} \\
& \geq \frac{1}{2} \min \left\{n, \operatorname{rank} E_{n} X E_{n}\right\} .
\end{aligned}
$$


Since each individual interval $E_{n}$ appears in the sequence infinitely many times, in fact rank $E_{n} Y E_{n} \geq \frac{1}{2}$ rank $E_{n} X E_{n}$. Finally, if $E$ is an interval of $\mathcal{N}$, let $E_{n}$ increase to $E$. Then rank $E_{n} Y E_{n}$ and rank $E_{n} X E_{n}$ increase to rank $E Y E$ and rank $E X E$ respectively, so we are done.

In the final example of the paper, we show how the norm closed ideal generated by certain diagonal operators can be related in a moderately satisfactory way to another natural object in the algebra.

Example 3.6. Let $\mathcal{H}$ be a Hilbert space. For vectors $x, y \in \mathcal{H}$, let $x y^{*}$ denote the rank-one operator $z \mapsto\langle z, y\rangle$. Suppose $\left\{e_{i}\right\}_{i=1}^{\infty}$ is an orthonormal basis for $\mathcal{H}$, let $E_{i}=e_{i} e_{i}^{*}$, set $P_{n}:=\sum_{1}^{n} E_{i}$ and let $\mathcal{N}$ be the nest contained in $B(\mathcal{H})$ whose elements consist of 0 , the identity, and the projections $P_{n}$. It is well known that the weak closure of the span of the elementary tensors, $(\operatorname{Alg} \mathcal{N}) \bar{\otimes} B(\mathcal{H})$, is a nest algebra. (The invariant projections are $P_{n} \otimes I$.) Let $(\mathrm{Alg} \mathcal{N}) \otimes \mathcal{K}(\mathcal{H})$ be the norm-closed span of the elementary tensors. Viewing $(\operatorname{Alg} \mathcal{N}) \otimes B(\mathcal{H})$ and $(\operatorname{Alg} \mathcal{N}) \otimes \mathcal{K}(\mathcal{H})$ as upper-triangular matricies with operator entries, one might expect that $(A \lg \mathcal{N}) \otimes \mathcal{K}(\mathcal{H})$ is an ideal in $\operatorname{Alg} \mathcal{N} \bar{\otimes}(\mathcal{H})$. However, it is not. Indeed, let

$$
Q:=I \otimes E_{1} \quad \text { and } \quad T:=\sum_{i=1}^{\infty} E_{i} \otimes e_{i} e_{1}^{*} .
$$

It is readily seen that $Q \in(\operatorname{Alg} \mathcal{N}) \otimes \mathcal{K}(\mathcal{H})$ and $T \in(\operatorname{Alg} \mathcal{N}) \otimes \mathcal{B}(\mathcal{H})$. Since the algebraic tensor product of $\operatorname{Alg} \mathcal{N}$ with the finite rank operators is norm-dense in $(\mathrm{Alg} \mathcal{N}) \otimes \mathcal{K}(\mathcal{H})$, we find that $T \notin(\operatorname{Alg} \mathcal{N}) \otimes \mathcal{K}$. But $T=T Q$; hence $(\operatorname{Alg} \mathcal{N}) \otimes \mathcal{K}(\mathcal{H})$ is not an ideal.

The following proposition shows that the closed ideal generated by $Q$ and the closed ideal generated by $\operatorname{Alg} \mathcal{N} \otimes \mathcal{K}$ are the same. In fact, slightly more is true.

Proposition 3.7. Let $\mathcal{I}$ be the closed ideal of $(\operatorname{Alg} \mathcal{N}) \bar{\otimes} \mathcal{B}(\mathcal{H})$ generated by $(\mathrm{Alg} \mathcal{N}) \otimes$ $\mathcal{K}(\mathcal{H})$ and let $K \in \mathcal{B}(\mathcal{H})$ be a non-zero compact operator. If $\mathcal{J}_{K}$ is the closed ideal of $(\mathrm{Alg} \mathcal{N}) \bar{B}(\mathcal{H})$ generated by $I \otimes K$, then $\mathcal{I}=\mathcal{J}_{K}$.

Proof. We first show that the ideals $\mathcal{J}:=\mathcal{J}_{E_{1}}$ and $\mathcal{I}$ coincide. Clearly $\mathcal{J} \subseteq \mathcal{I}$. To obtain the reverse inclusion, note that for any finite rank operator $F \in B(\mathcal{H})$ and any $A \in \operatorname{Alg} \mathcal{N}, A \otimes F$ satisfies the hypotheses of Theorem 3.1, and so an application of that theorem shows $A \otimes F \in \mathcal{J}$. Taking the closure of the span of such operators, we conclude that $(\operatorname{Alg} \mathcal{N}) \otimes \mathcal{K}(\mathcal{H}) \subset \mathcal{J}$. Hence $\mathcal{I}=\mathcal{J}$.

Now let $K$ be an arbitrary non-zero compact operator. Again, it is clear that $\mathcal{J}_{K} \subseteq \mathcal{I}$. Choose an operator $S$ so that $K S=E_{1}$. Then $I \otimes E_{1}=(I \otimes K)(I \otimes S)$, so $I \otimes E_{1} \in \mathcal{J}_{\mathcal{K}}$ and hence $\mathcal{I}=\mathcal{J}_{E_{1}} \subseteq \mathcal{J}_{K}$. 


\section{REFERENCES}

1. W. B. ARveson, Interpolation problems in nest algebras, J. Funct. Anal. 20 (1975), 208-233.

2. K. R. Davidson, Nest Algebras (Pitman Research Notes in Mathematics Series, 191, Longman Scientific and Technical Pub. Co., London, New York, 1988).

3. K. R. Davidson, K. Harrison and J. L. OrR, Epimorphisms of nest algebras, Internat. J. Math., to appear.

4. J. A. Erdos and S. C. Power, Weakly closed ideals of nest algebras, J. Operator Theory 7 (1982), 219-235.

5. D. R. Larson and D. R. Pitrs, Idempotents in Nest Algebras, J. Funct. Anal. 97 (1991), 162-193.

6. J. L. ORR, The maximal ideals of a nest algebra, J. Funct. Anal. 124 (1994), 119-134.

7. J. L. ORR, Automorphism invariant ideals of a continuous nest algebra, preprint.

Mathematics Department

UNIVERSITY OF NEBRASKA

LINCOLN, NE 68588

USA

E-mail address: jorr@unlinfo.unl.edu
Mathematics Department

UNIVERSITY OF NEBRASKA

LINCOLN, NE 68588

USA

E-mail address: dpitts@unlinfo.unl.edu 